\title{
Réflexions sur l'utilisation des longues séries d'observations climatologiques dans le cadre de l'étude du climat et de son évolution
}

\author{
D. Duband \\ EDF - Division technique générale \\ 37, rue Diderot - B.P. 41 - Grenoble Cedex
}

\begin{abstract}
Depuis quelques années, le grand public, les hommes politiques, les gouvernements des pays industrialisés ont été largement sensibilisés à l'évolution du climat par l'intermédiaire des médias qui se faisaient l'écho et l'amplificateur des résultats et hypothèses de recherches scientifiques.

Cette information qui a pour intérêt principal de faire prendre conscience de l'interaction croissante entre les activités de l'homme et son environnement continental, aquatique, glaciaire, océanique et atmosphérique, doit cependant rester objective si elle veut rester crédible; ceci sans verser dans le catastrophisme.

Il est évident que l'étude précise et fine entreprise sur l'évolution du climat passé est fondamentale pour comprendre son état actuel et élaborer des scenarii pour le futur; tout cela en essayant de distinguer la part qui peut revenir à l'homme dont la croissance démographique s'amplifie depuis 200 ans et particulièrement depuis 1950 .

Comment discriminer d'après 100 ans d'observations les aléas normaux d'un climat stable des prémisses éventuels
\end{abstract} d'une rupture climatique brutale, due à l'homme ou à une
cause qui le dépasse?

Les échelles de temps (un million, cent mille, mille, cent années)

La terre a plus de 4 milliards d'années d'existence. Au cours de cette période elle a connu de grands bouleversements géologiques et climatiques. Cette histoire de la terre dont on précise progressivement les jalons temporels c'est d'abord aux géologues et aux glaciologues que nous en sommes redevables puis à d'autres spécialistes climatologues, palynologues, paleantologues, dendroclimatologues, météorologues, chimistes, astronomes etc... [8, 11].

Ces chercheurs ont établi que les calottes glaciaires permanentes existent depuis plusieurs millions d'années, la terre ayant connu d'autres périodes de glaciation dans un

\section{Reflections on the use of long series of climatological observations} as part of the study of climate and its changes

Over the last few years, the general public, politicians and governments of the industrialized countries have become very aware of changes in climate through the media which have been reporting and amplifying the results and hypotheses of scientific research. The main interest of this information is to heighten awareness of the growing interaction between mans' activities and his continental, aquatic, glacial, oceanic and atmospheric environment, yet it must remain objective if it is to remain credible; this without plunging into sensational doomsday type stories. It is obvious that the precise and fine study undertaken on past changes in climate is fundamental in order to understand its present state and draw up possible scenarios for the future; all this in trying to take into account the effect of man with increased demographic growth over the last 200 years and in particular since 1950. How can one discern from 100 years of observations of the normal movements of a stable climate, possible premises of a brutal climatic break due to man or to a cause that is beyond him? 
passé plus lointain. Puis ils ont mis en évidence que depuis cinq cent mille ans la terre avait connu cinq glaciations et déglaciations, une tous les cent mille ans, la principale cause étant l'énergie solaire.

L'analyse fine de carottages de glace effectuées en arctique et antarctique a révélé l'histoire de la dernière glaciation terrestre il y a 18000 ans et la déglaciation qui a suivi. Ainsi depuis moins de trois mille ans nous sommes soumis à un climat à peu près stable ce qui ne veut pas dire uniforme ni stationnaire. Et nous disposons de quelques séries de mesure de paramètres climatologiques ponctuels comme la température de l'air, la précipitation, la pression atmosphérique, au mieux depuis 250 ans (la plupart des instruments ont été inventés par les physiciens depuis un peu plus de 300 ans, sauf le pluviomètre qui existe depuis plus de 1000 ans).

En fait, en Europe, les "réseaux» de mesure ont commencé à fonctionner après 1800 , mais il faut attendre 1860 pour avoir une densité spatiale raisonnable soit un peu plus de 100 ans.

\section{Importance et qualité des chroniques historiques}

Il s'agit des mesures de précipitation, température de l'air, pression atmosphérique au sol, débits, niveau de lac $[1,2$, 4, 6, 7].

Bien que l'on ne dispose en Europe que d'un nombre limité de séries de mesure depuis près de 150 ans, et parfois 250 ans grâce à nos amis suisses, un inventaire exhaustif reste à faire dans les pays européens, en particulier pour la période 1850-1900. C'est d'ailleurs le projet du groupe de travail sur les données pluviométriques anciennes suscité en France, par le Ministère de l'Environnement et la Météorologie nationale.

Il est important pour apprécier l'éventualité des aléas et fluctuations (valeurs extrêmes, moyennes, séquences...) inscrites dans la potentialité d'un climat stable de disposer non seulement de longues séries de données journalières, mensuelles, annuelles, mais d'avoir une bonne évaluation de leur extension spatiale en s'appuyant sur les réseaux de mesure relativement étoffés.

Une fois localisés et identifiés les sites disposant d'informations historiques de longue duree, une action indispensable consiste à contrôler, critiquer, éventuellement reconstituer les données manquantes de ces chroniques d'observations et les valider. Cet énorme travail a pour objectif de filtrer les effets de déplacements de stationchangement de capteur - modification de la procédure de la mesure, modification de l'environnement de la station (ex.: effet de l'urbanisation sur l'augmentation de la température de l'air).

Il devrait y avoir dans chaque pays un comité de validation et qualification des séries chronologiques, pour chacune des stations où des observations historiques ont été effectuées: un "observatoire des chroniques anciennes " pour permettre aux chercheurs et aux scientifiques d'utiliser une base d'informations fiables et validées.
Peut-on identifier un signal climatique déterminant à l'échelle de 100 ans parmi les "bruits " des fluctuations « normales »

C'est une des grandes questions actuelles et sujette à controverse. En effet, certains scientifiques pensent que l'on peut détecter sur quelques dizaines d'années les risques avant coureurs d'une modification irréversible du climat, alors que d'autres pensent que l'inertie et les effets interactifs/rétroactifs des composantes de l'écosystème terrestre (atmosphère - hydrosphère - cryosphère - biosphère - continents) se diffusent sur plusieurs centaines d'années.

On peut d'ailleurs observer sur une chronique historique, pendant quelques années, l'accroissement ou la diminution d'un paramètre qui semble annoncer une tendance, puis constater ensuite sa décroissance ou croissance d'où une pseudo période.

En ce qui nous concerne, l'analyse (locale et spatiale) de quelques dizaines de longues séries historiques en France et en Suisse de mesure de précipitations et de températures de l'air, qui sont des témoins importants du cycle de l'eau et donc des variations du climat, ne nous a pas permis d'identifier de tendance significative (fig. $l$ à 26, in fine).

A l'échelle mensuelle et annuelle, il y a une étroite corrélation entre les séries de températures de l'air mesurées en France et en Suisse en plaine telles que Bâle, Genève, Clermont-Ferrand, Pau, Gap, de même qu'il y a une étroite corrélation entre les séries de températures mesurées en altitude (au-dessus de $1400 \mathrm{~m}$ ) telles que le Puy de Dôme, le Grand Saint Bernard, le Mont Aigoual et le Pic du Midi.

Cette corrélation spatiale a permis de déceler une anomalie dans la série bicentenaire de Bâle pour la période 1961-1988. Les stations d'altitude sont précieuses car non influencées par les effets d'urbanisation, on regrettera à cette occasion l'arrêt des mesures au Pic du Midi et au Puy de Dôme.

Les corrélations spatiales établies pour la période 18901980 permettent de valoriser l'information temporelle fournie par les observations de Bâle, Genève depuis 1755 et du Grand Saint Bernard depuis 1818.

Pour ce qui concerne les précipitations, on ne dispose pas d'aussi longues séries historiques que pour la température. La corrélation spatiale entre mesures n'est pas aussi élevée mais depuis le début de ce siècle on dispose quand même d'une base de données utilisable et qui devrait pouvoir s'étoffer [4].

L'absence de tendance est également la conclusion à laquelle aboutit une équipe de chercheurs américains du NOAA ayant effectué en 1989 une analyse numérique et statistique approfondie sur plus de 6000 séries de stations pluviométriques et thermométriques américaines de plus de 90 ans (1895-1987) [6]. Cette étude remet en cause les conclusions un peu hatives d'une équipe de la NASA effectuée antérieurement sur les mêmes données non filtrées [5].

Un autre chercheur travaillant sur de très longues observations hydrologiques historiques de débits et niveaux de 
lac aux Etats-Unis et en Afrique, en arrive à la même conclusion [10].

La Météorologie nationale a également adopté une attitude très prudente et circonspecte dans ses analyses climatologiques [13]. Ces résultats contredisent certaines affirmations d'auteurs anglais comme John, et qui ont d'ailleurs été cités comme référence par des chercheurs français [9].

Il me semble primordial de lever d'abord toutes les contradictions issues de l'analyse des séries climatologiques historiques avant d'aller plus avant dans les hypothèses de modifications climatiques actuelles $[5,6]$.

A-t-on vraiment exploré toute la potentialité du climat contemporain en évènements rares : sécheresses de 19891949-1921... vagues de froid de 1985-1963-1956-1942-1880, crues catastrophiques de $1856-1910 \ldots$ ?

Il serait d'ailleurs très intéressant d'expliquer les accidents climatiques au moins Européens tels que la période de redoux du $\mathrm{IX}^{\mathrm{C}}$ au XIII ${ }^{\mathrm{c}}$ siècle, qui favorisa la migration des Vikings et le petit âge glaciaire du XVI ${ }^{e}$ au XVIII ${ }^{\mathrm{e}}$ [12].

\section{L'extrapolation du taux de $\mathrm{CO}_{2}$, dans le futur}

Il est indéniable que le taux de $\mathrm{CO}_{2}$ dans l'atmosphère augmente de façon significative et continue depuis le début de ce siècle avec toutefois des paliers qu'on n'explique pas, actuellement. Il en va de même du méthane et d'autres composés. Bien que ce ne soient pas les seuls acteurs ou ingrédients d'un effet de serre possible puisqu'il y a également la vapeur d'eau.

Il existe peu de sites de mesure du $\mathrm{CO}_{2}$ anciens avec des observations quotidiennes : la série historique de référence depuis 1958, Mauna Loa, est située à Hawai ; de nombreuses stations ont cependant été créées depuis dans différents pays du globe.

Sur l'origine du $\mathrm{CO}_{2}$, il n'y a pas de véritable consensus : certains privilégient les éruptions volcaniques [3], le mouvement des plaques tectoniques, la déforestation, d'autres au contraire les combustibles fossiles (Centrales à charbon et à fuel, voitures...). La consommation mondiale d'énergie augmente régulièrement.

Pour les tenants de cette dernière hypothèse on conçoit que les extrapolations de taux de $\mathrm{CO}_{2}$ dans l'atmosphère à l'an 2030 ou 2050 soient alarmistes et extrêmement préoccupantes puisqu'elles sont sous-tendues par les projections démographiques mondiales.

En effet, la population du globe terrestre qui était de 2,5 milliards d'habitants en 1950 , a atteint 5 milliards d'habitants en 1989, et les projections des instituts démographiques annoncent 8,5 milliards en 2025 (la fourchette allant de 7,5 à 9,5); l'augmentation de population provenant essentiellement des pays en voie de développement (Inde, Bengladesh, Chine, Pakistan, Indonésie, Brésil, Afrique).

C'est très probablement dans cette dimension que se trouvent les vrais problèmes du futur concernant l'environnement de la planète Terre.

\section{Conclusion}

Ces quelques réflexions n'ont pas la prétention de minimiser les recherches actuelles concernant les tenants et aboutissants du climat terrestre, car il faut être vigilants mais prudents et nous avons encore beaucoup à apprendre dans nos modélisations des processus terrestres et solaires qui interviennent dans le fonctionnement des climats.

Mais l'utilisation de nos modestes mais précieuses séries d'observations anciennes, effectuées durant ce siècle et le précédent, doit s'accompagner au préalable d'une grande rigueur quant à la qualification de ces données et des plus grandes précautions quant à leur interprétation, pour tenter de répondre à cette question fondamentale : l'homme peut-il modifier radicalement son environnement terrestre en quelques dizaines d'années ou la nature a-t-elle des ressources insoupçonnées pour imposer sa loi climatique.

\section{Références bibliographiques}

[1] Pekka Alemines. - Variations of the sea temperature around the coast of Finland. Conference on Climate and Water Helsinki 11/15 sept. 1989.

[2] J. BALEK. - Measurement of the historical changes of Aquatic environment under climatic impacts. Conference on Climate and Water - Helsinki 11/15 sept. 1989.

[3] Reid A. Bryson. - Volcans et Climats - La Recherche n' 135 - juillet-août 1982.

[4] H. F. Diaz, R. S. Bradley, J. K. Eischeid. - Precipitation - fluctuation over Global Land areas since Late 1800's Journal of Geophysical Research V. $94 \mathrm{n}^{\circ} 1$ - January 1989.

[5] J. HANSEN ans S. LEBEDEFF. - Global surface air temperatures update through 1987 - Geophysical Research Letters vol. $15, \mathrm{n}^{\circ} 4$, April 1988.

[6] Kirby Hanson, G. A. Maul, T. K. Karl. - Are Atmospheric " Greenhouse effects apparent in the climatic records of the contiguous US (1895-1987)" ? Geophysical Research Letters Vol. 16, n 1, January 1989.

[7] Raino Heino. - Changes of precipitation in Finland. Conference on Climate and Water - Helsinki 1989.

[8] J. Joseph JACQUET. - Les grands mouvements climatiques La Houille Blanche n 1-1990.

[9] Philip D. JONEs. - Le climat des mille dernières années. $L a$ Recherche $\mathrm{n}^{\circ} 219$ - Mars 1990.

[10] Geoff KiTE. - Use of time series analysis to detect climatic change. Journal of Hydrology 111 (1989) p. 259-279.

[11] J. LabeYrie. - L'homme et le climat - Denoël - Paris 1985.

[12] Emmanuel Leroy LAdURIE. - Histoire du climat depuis l'an Mil - Flammarion.

[13] Guy LaRIVIĖRE. - Il n'y a plus de saison... La Météorologie $\mathrm{n}^{\circ} 25,1988$. 


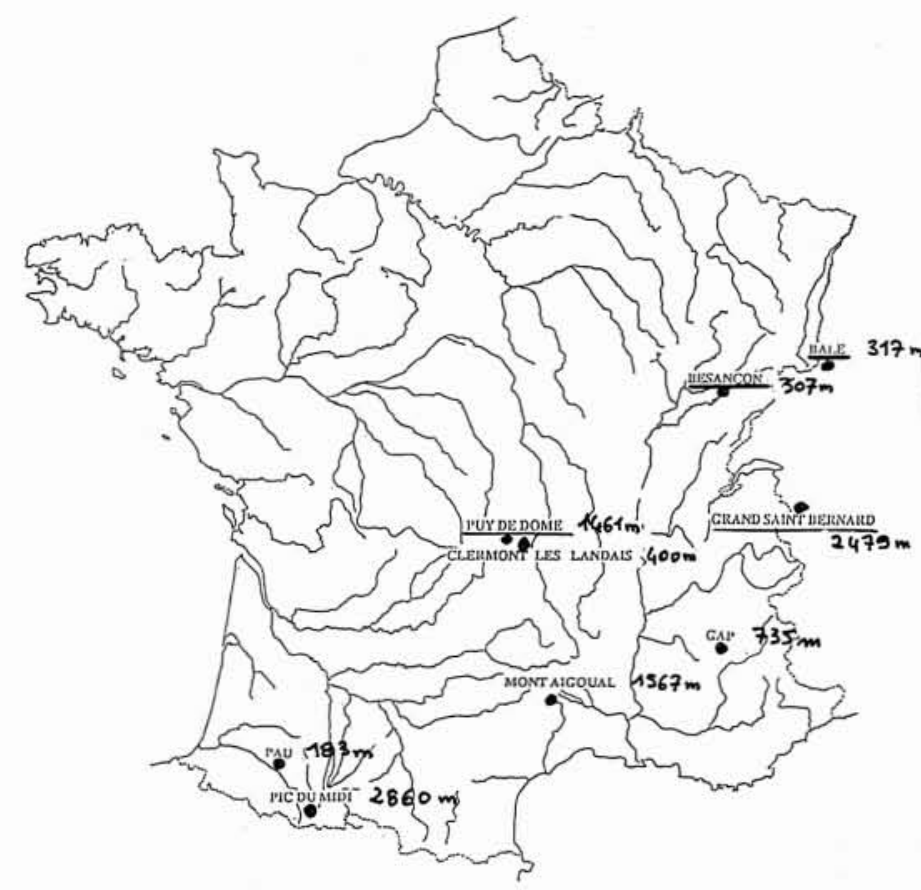

1. Stations de mesure des précipitations et des températures.
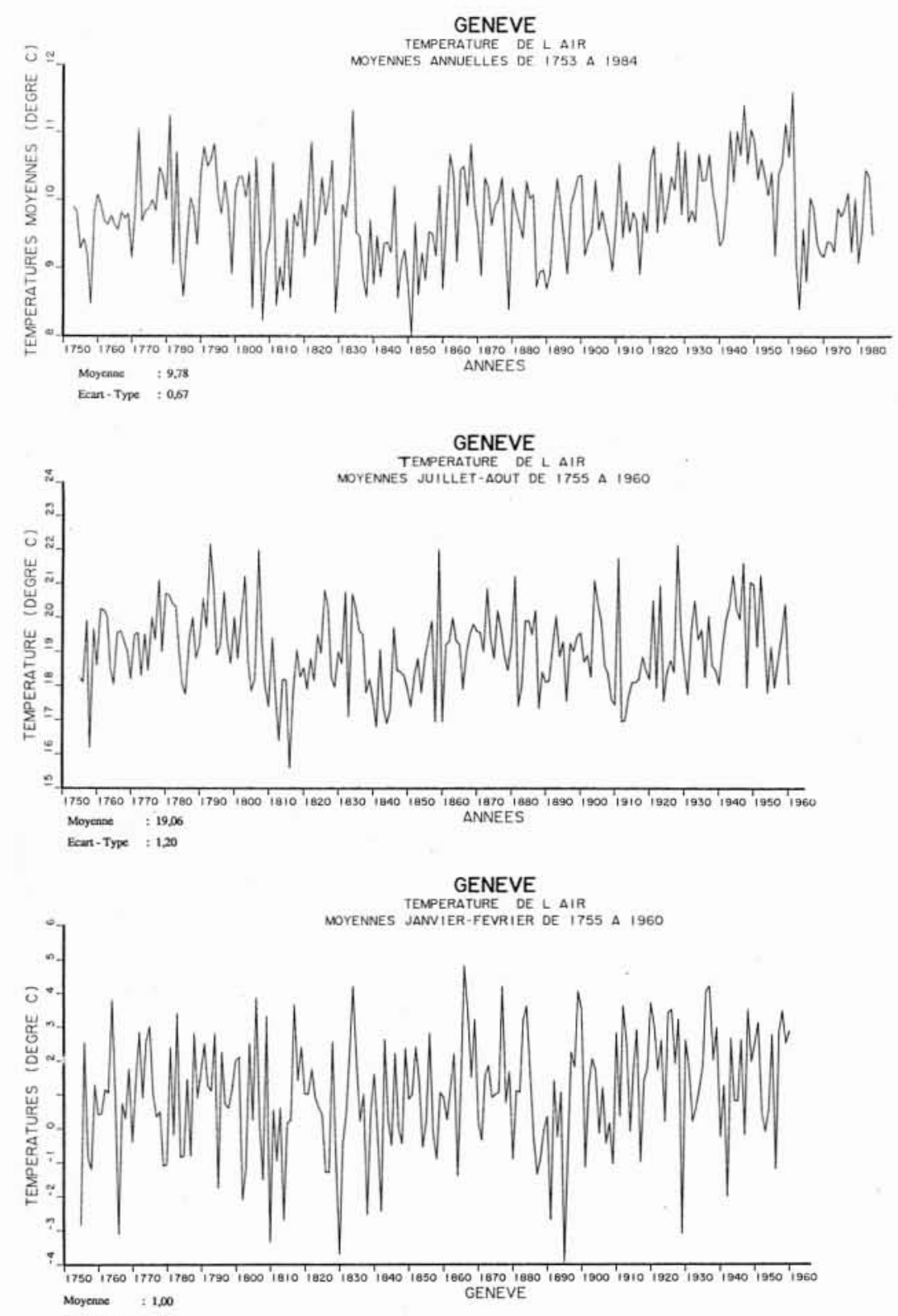

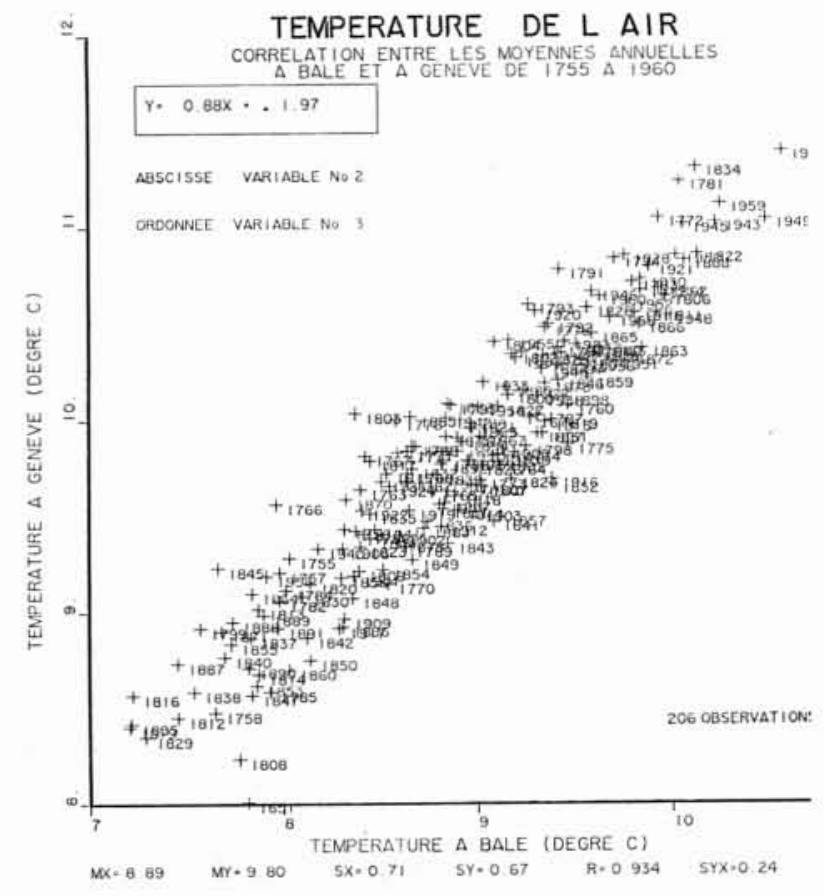

5.

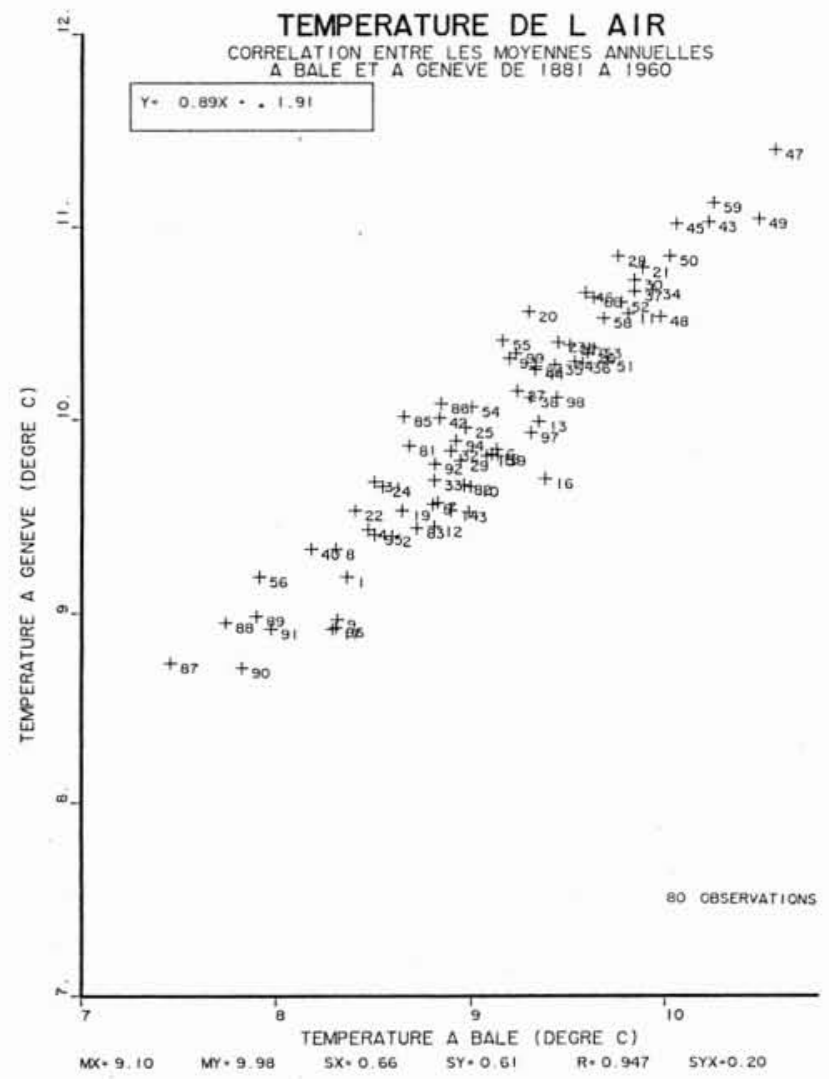

6. 

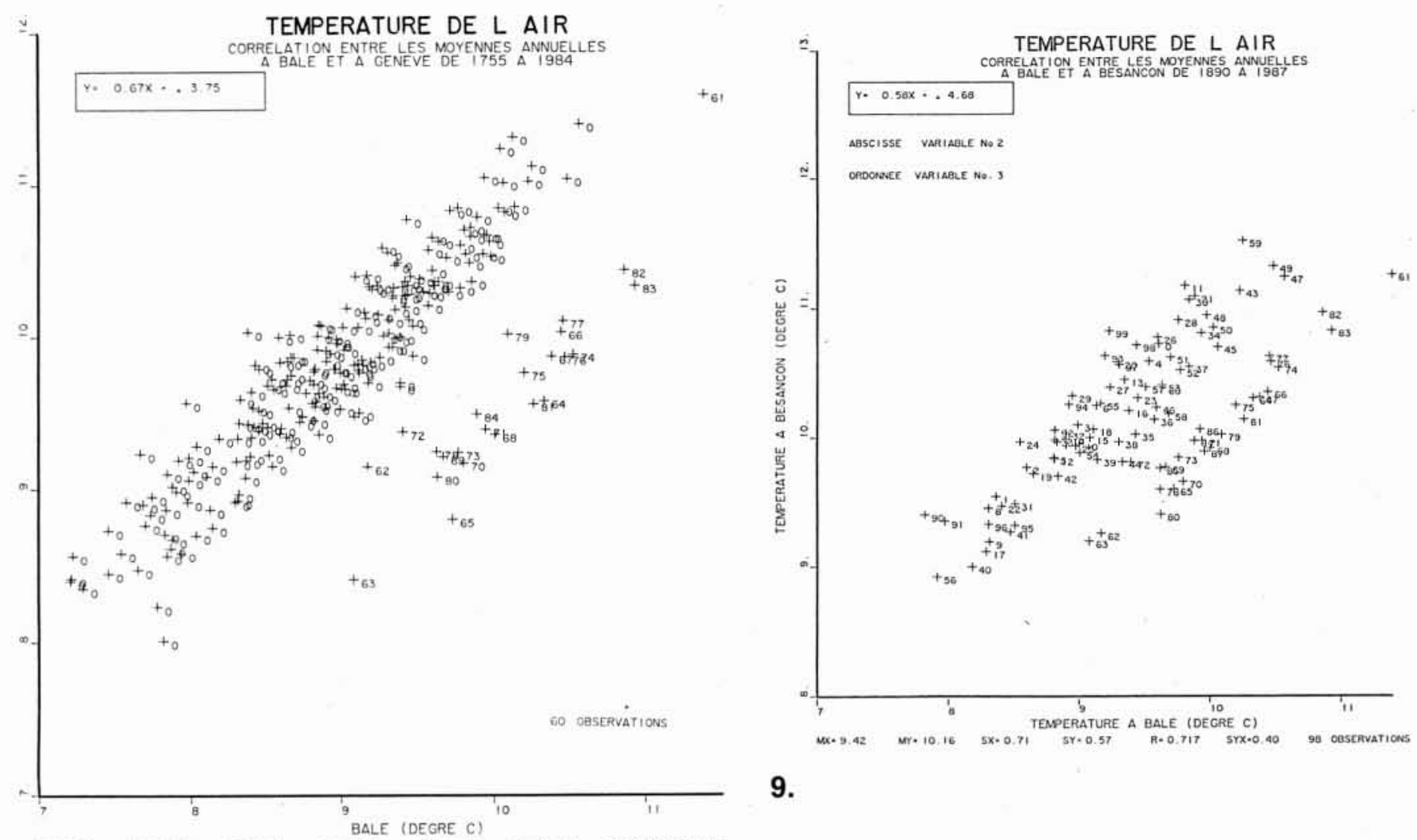

9.
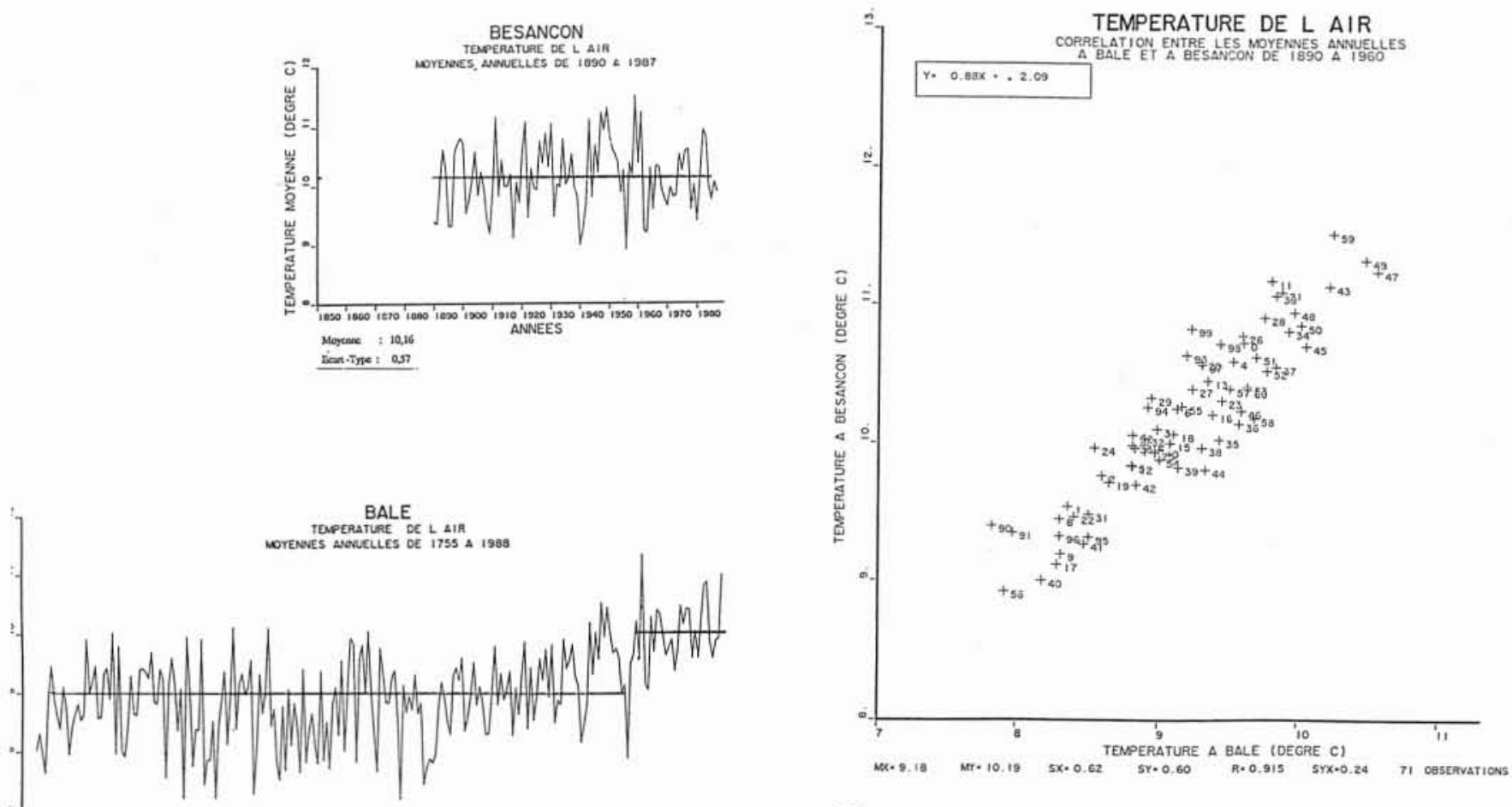

10. 


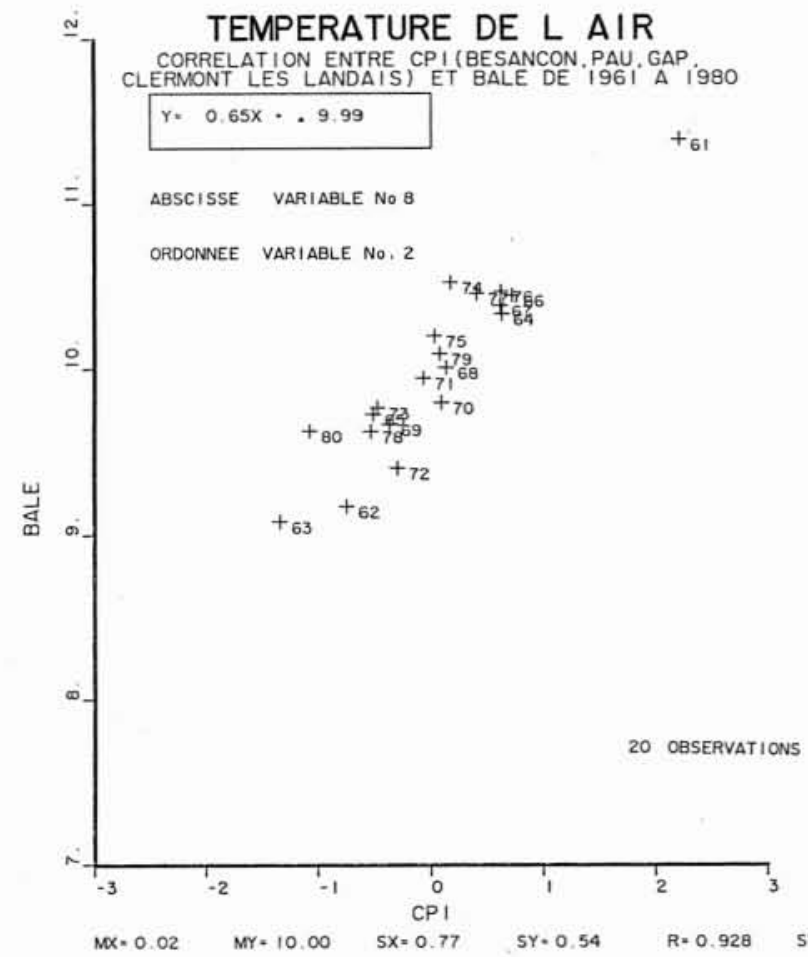

11.

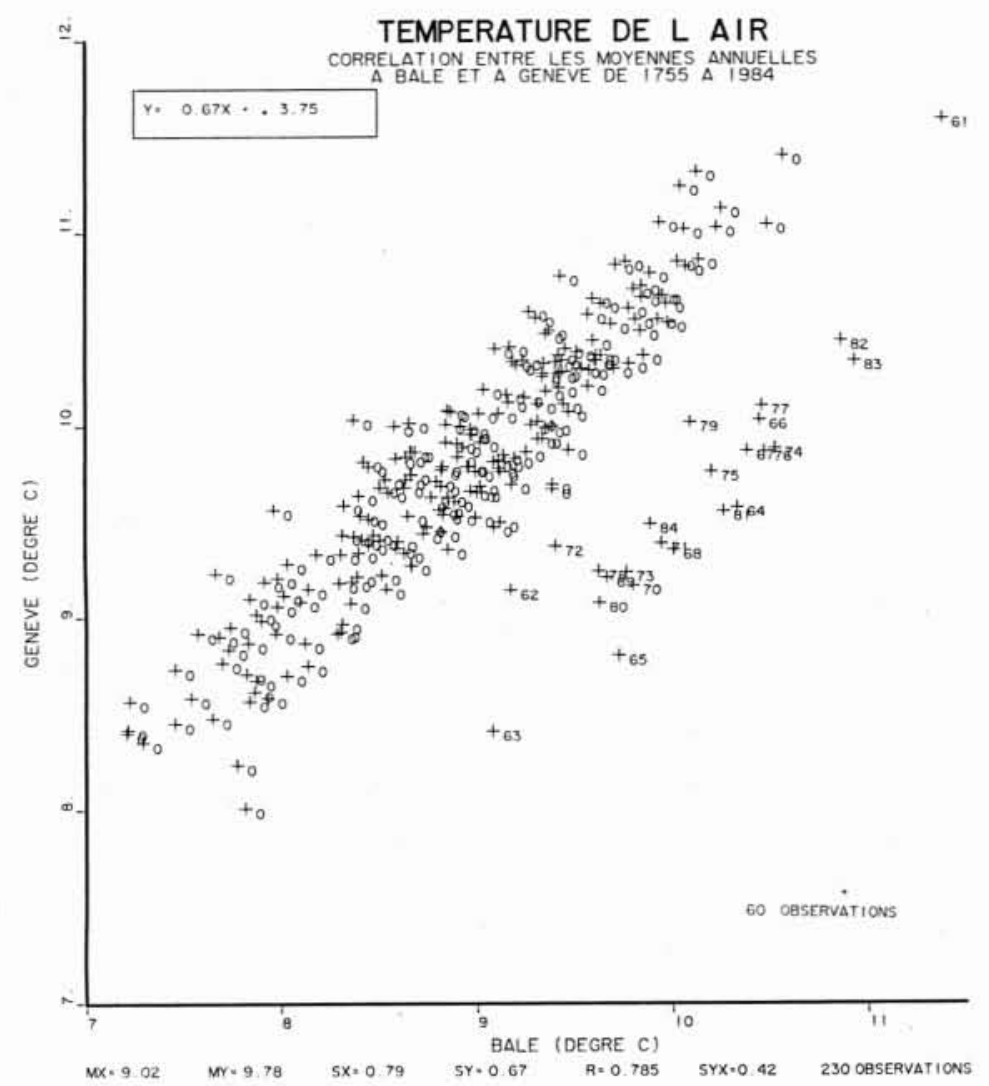

12.

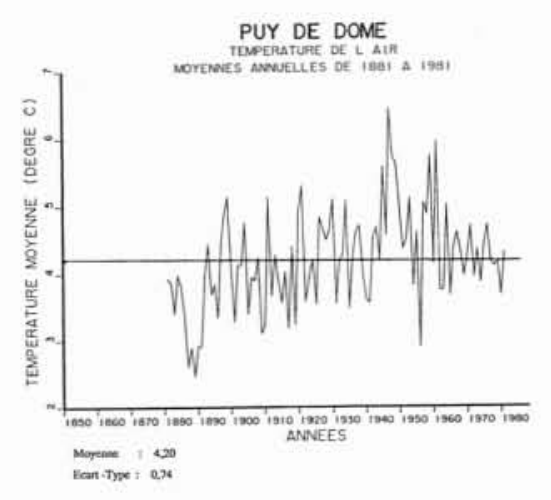

13.

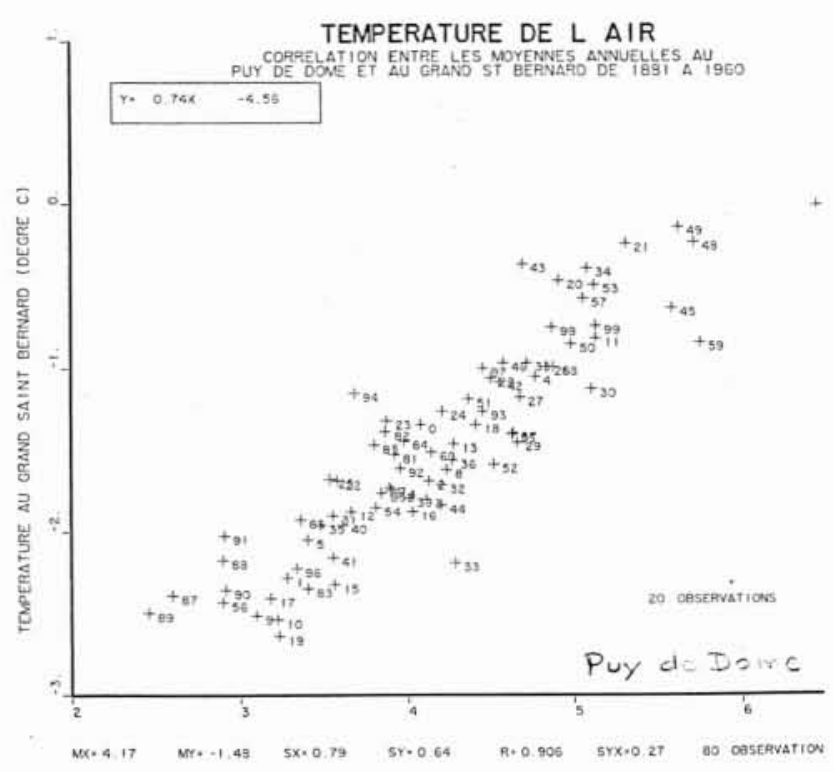

14.

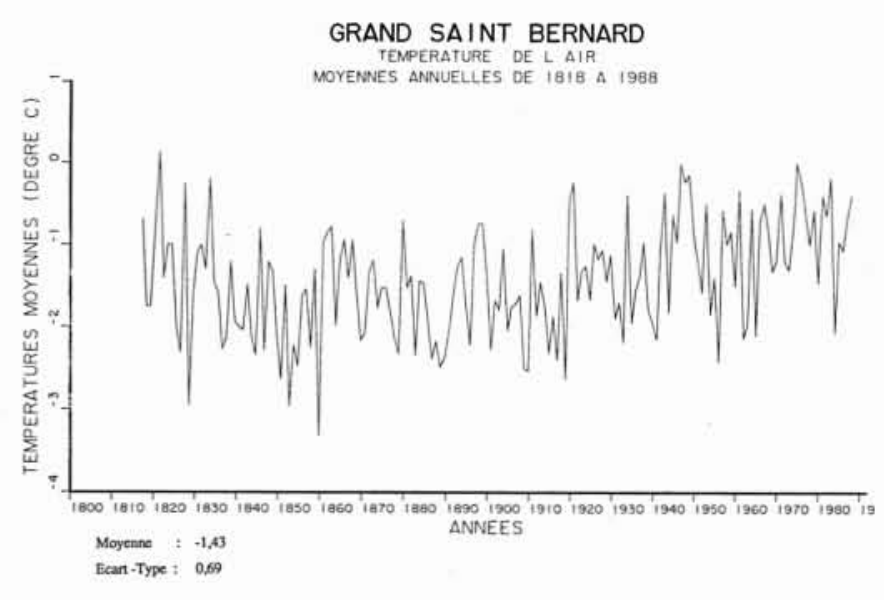

15. 


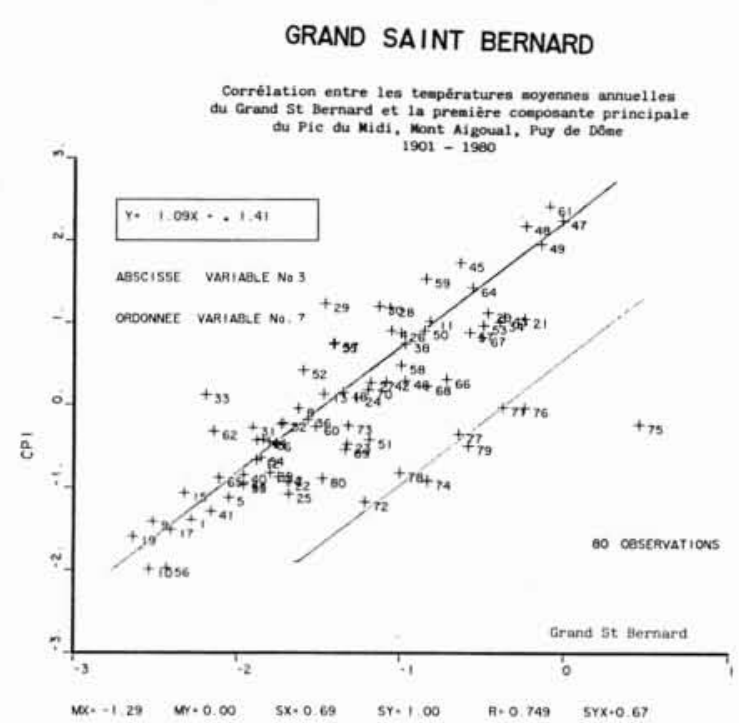

16.

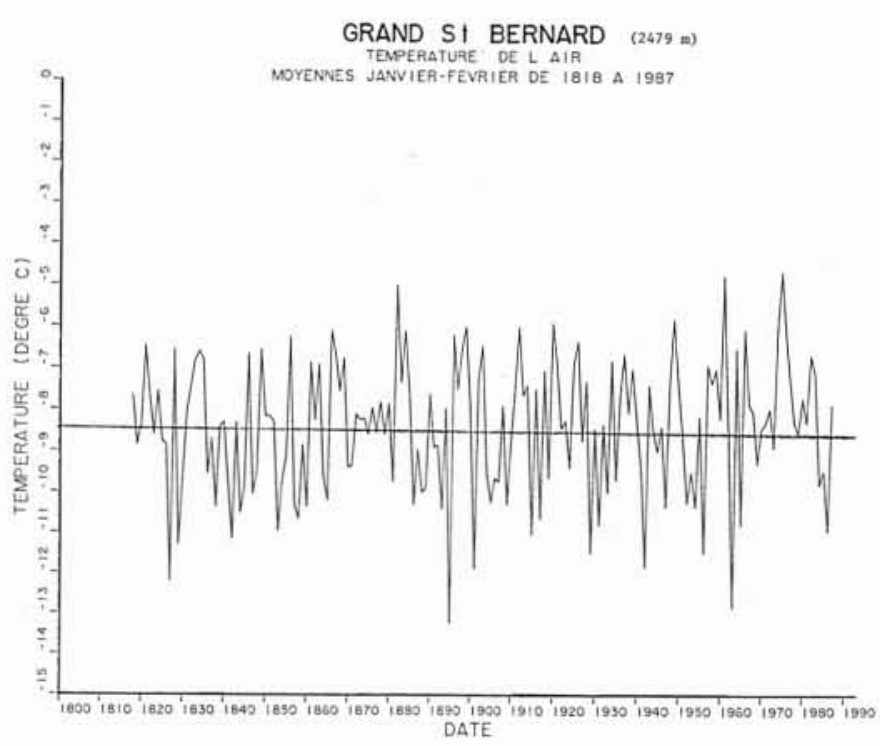

17.

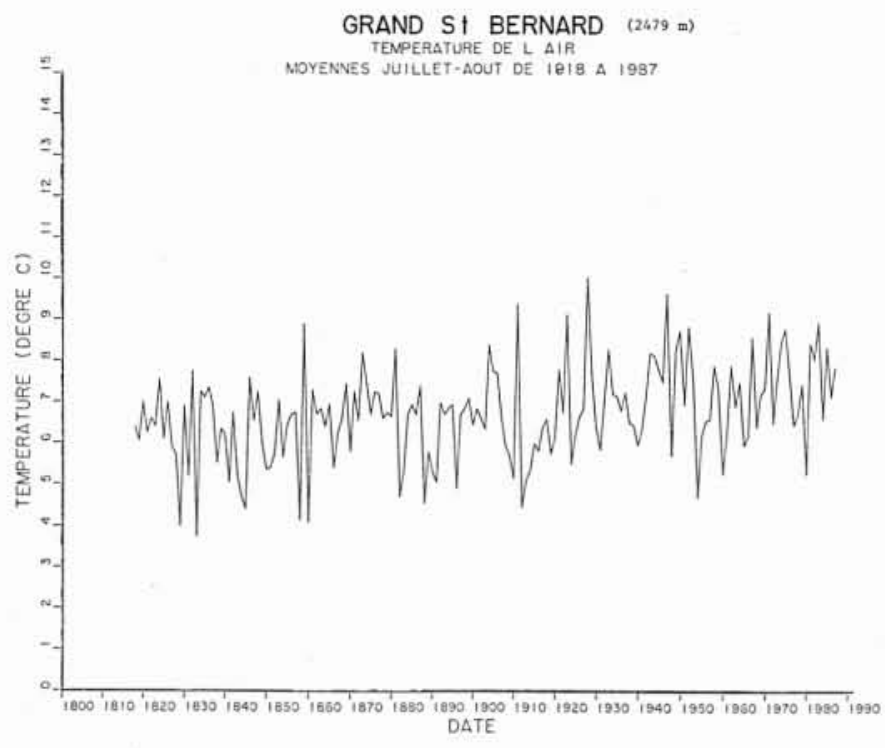

18.

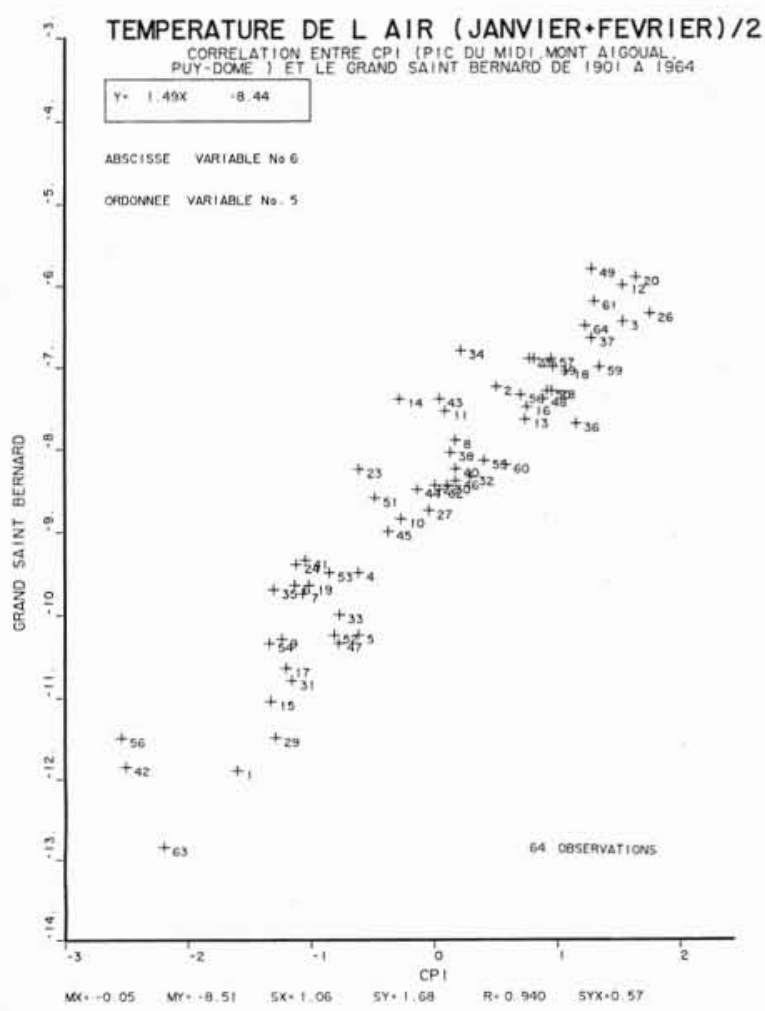

19. 

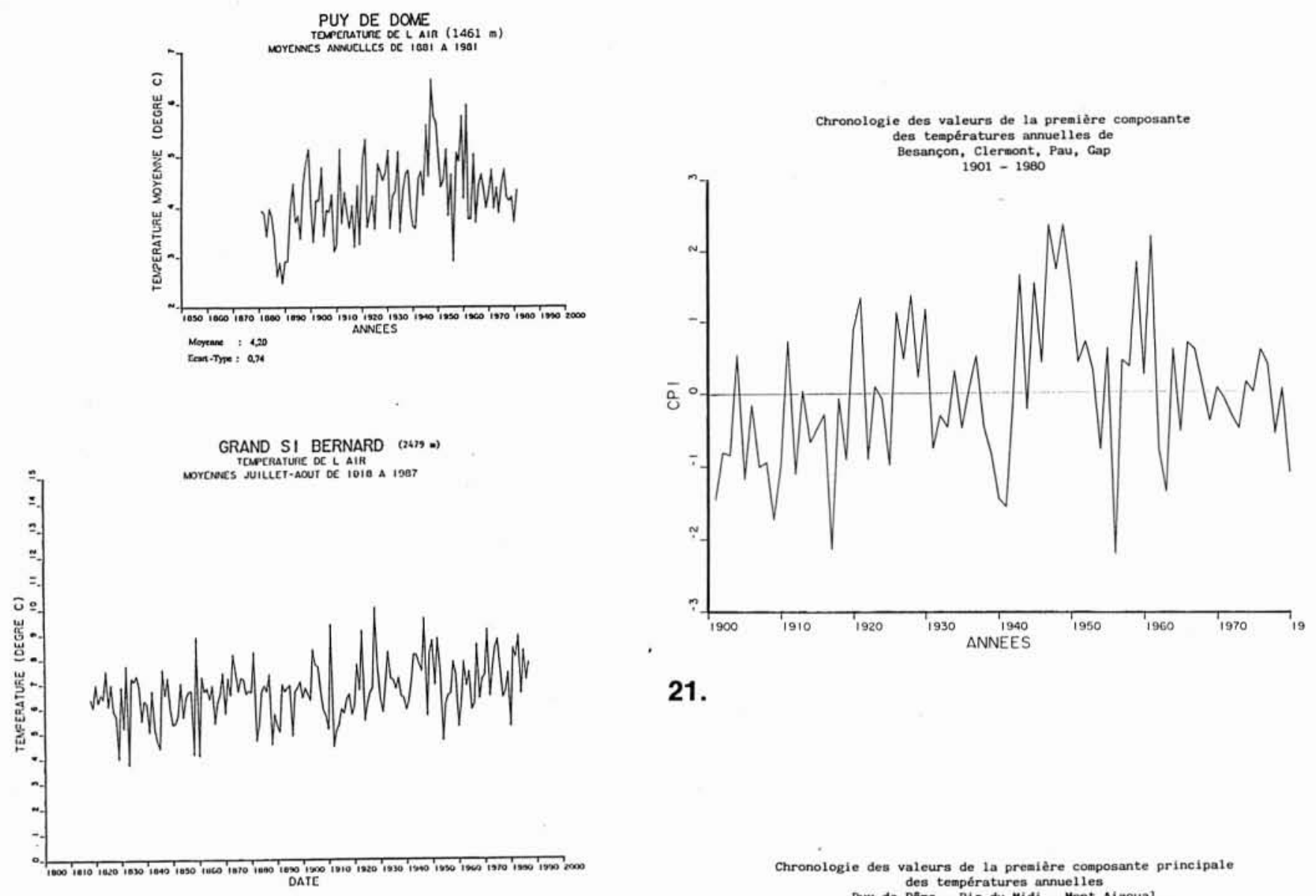

21.
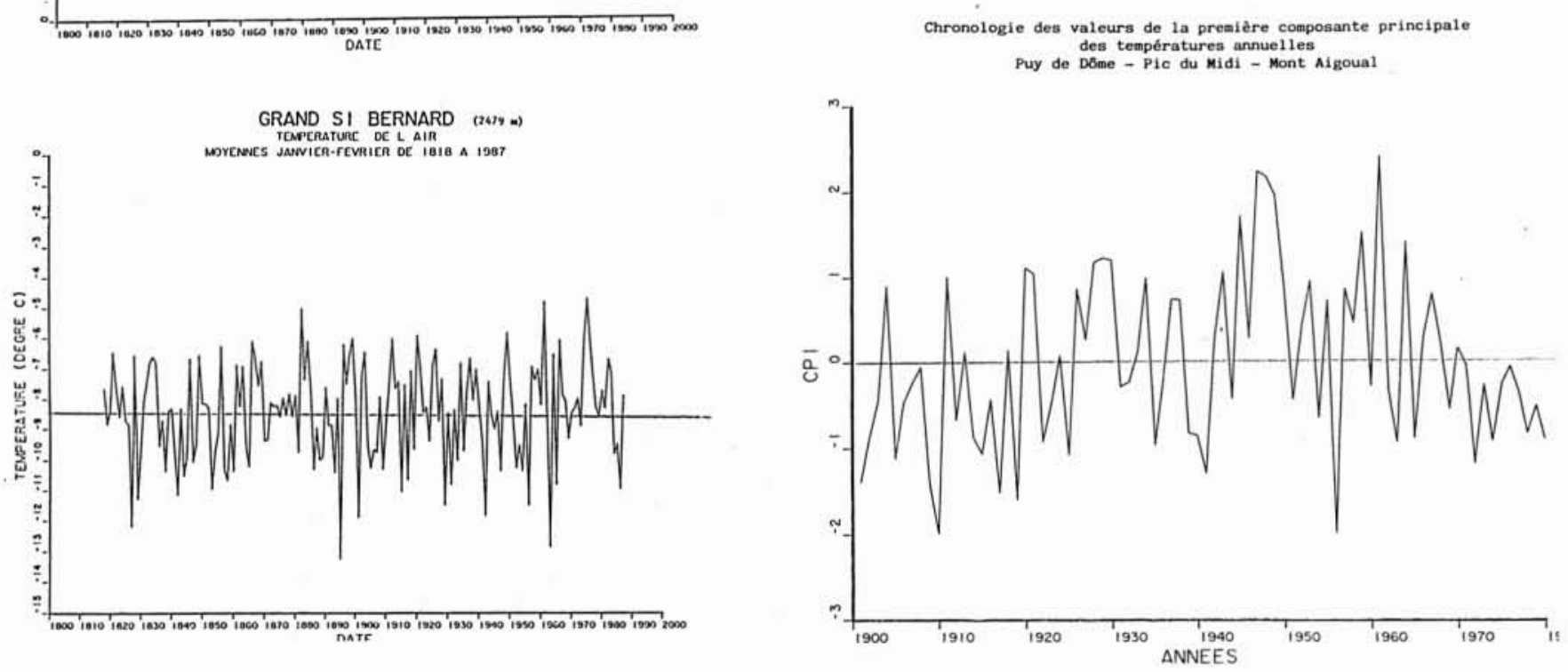

20.

22. 


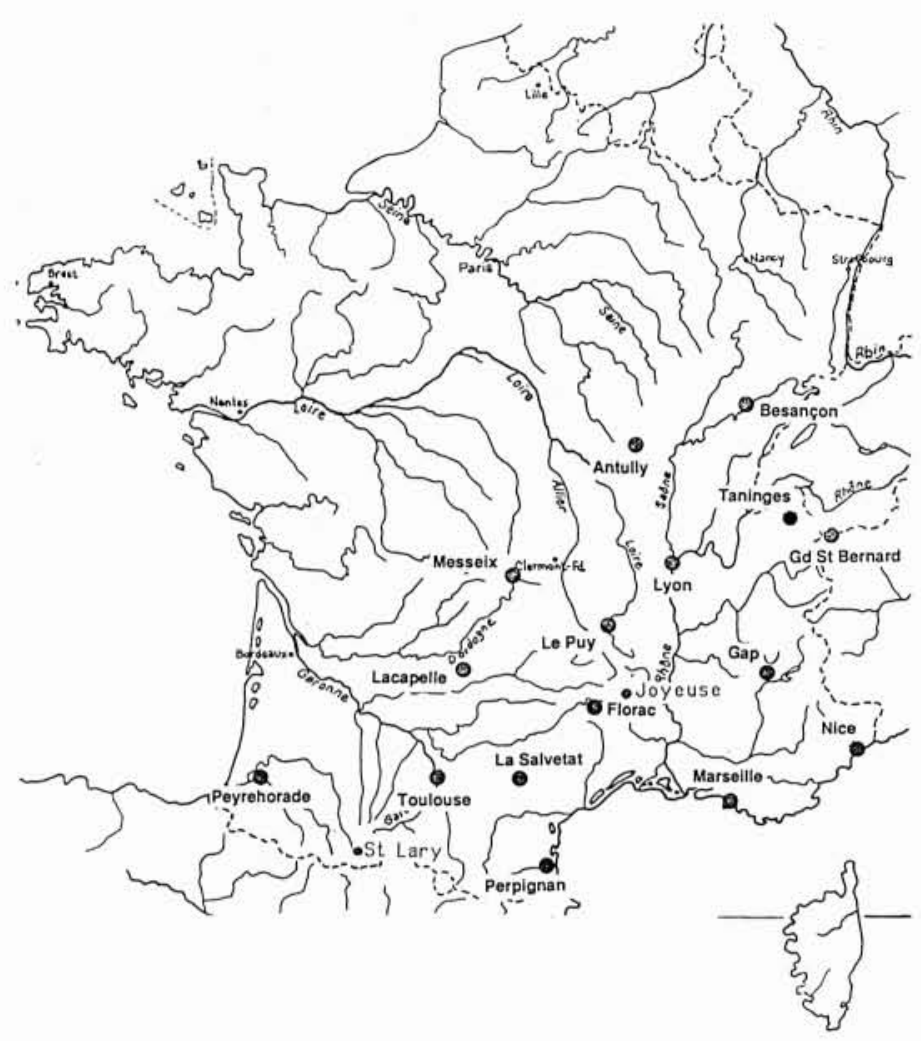

\section{LOCALISATION DES 16 STATIONS}

23. Localisation des 16 stations.

Chronologie des valeurs de la lère composante principale
des précipitations d'hiver, de 18 stations françaises, cumulées de Novembre à Avril, pour la période $1913-1988$

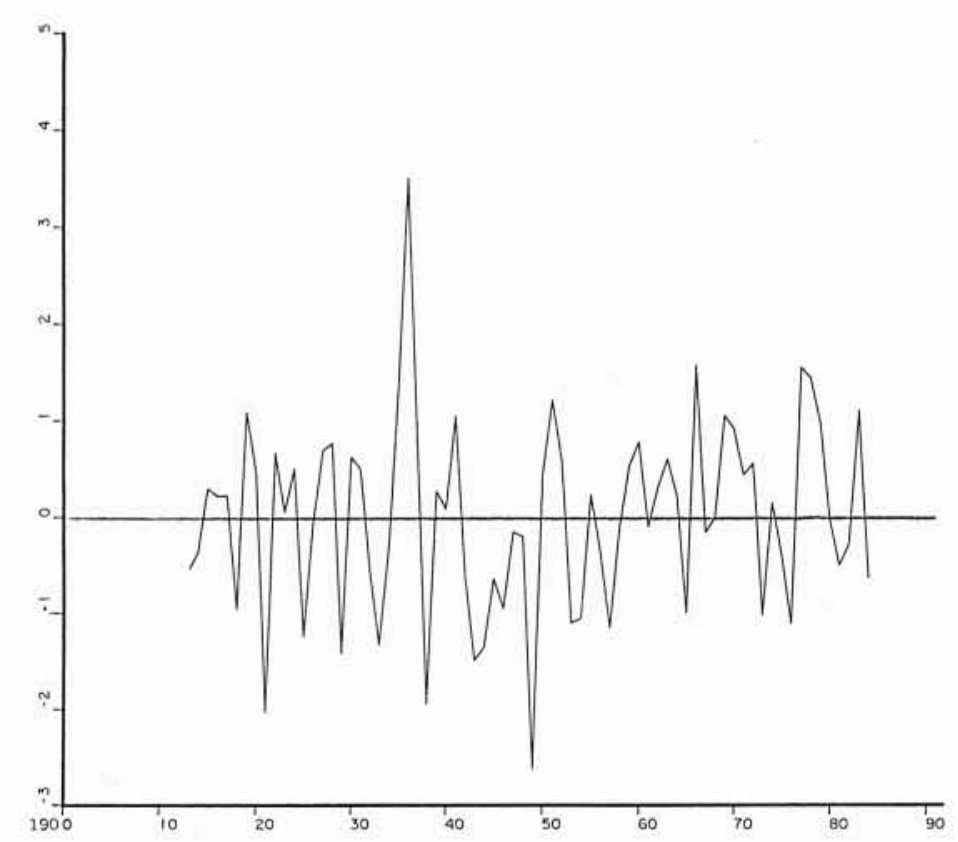

24.
SERIE DES PRECIPITATIONS ANNUELLES GAP (1861-1984)

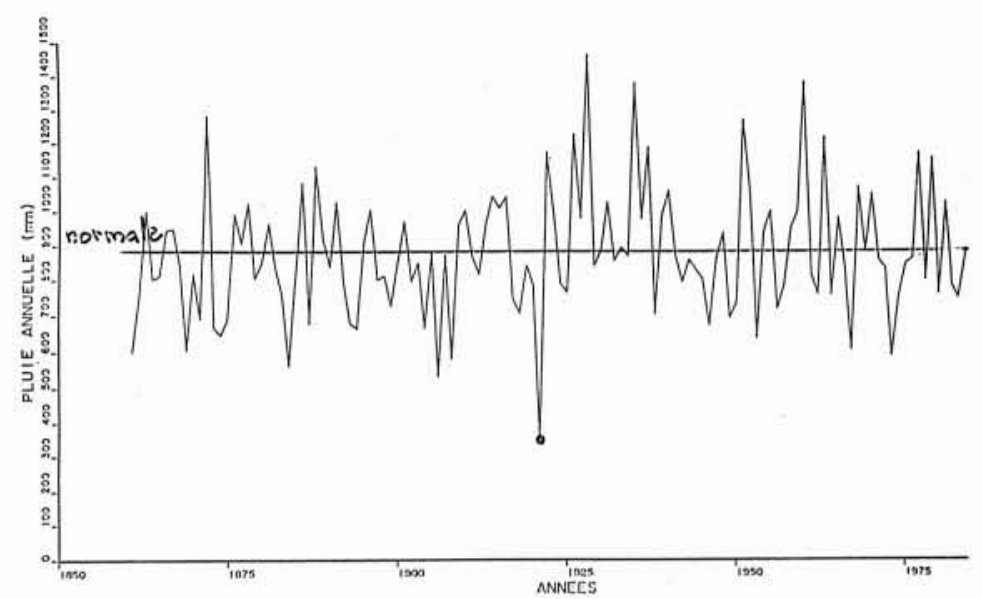

25.

COEFFICIENT DE PRODUCTIBILITE HYDRAULIQUE FRANCE
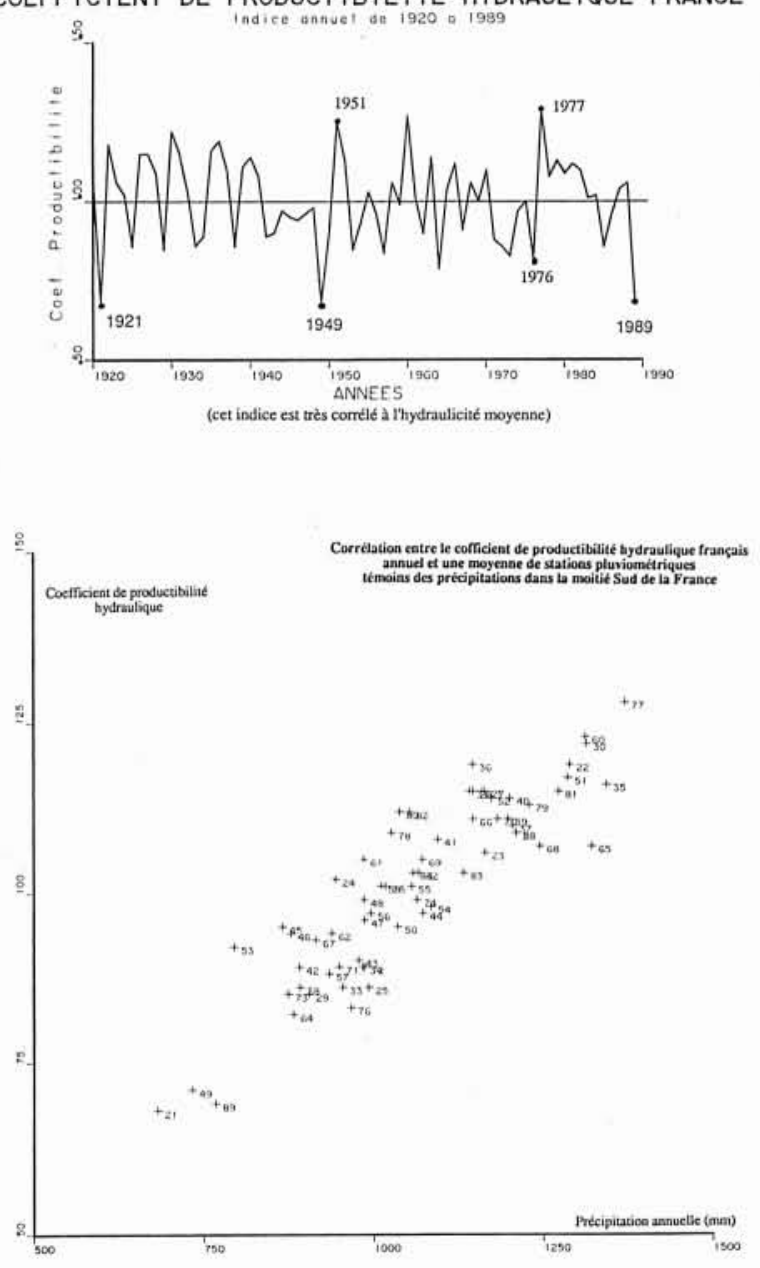

26. 


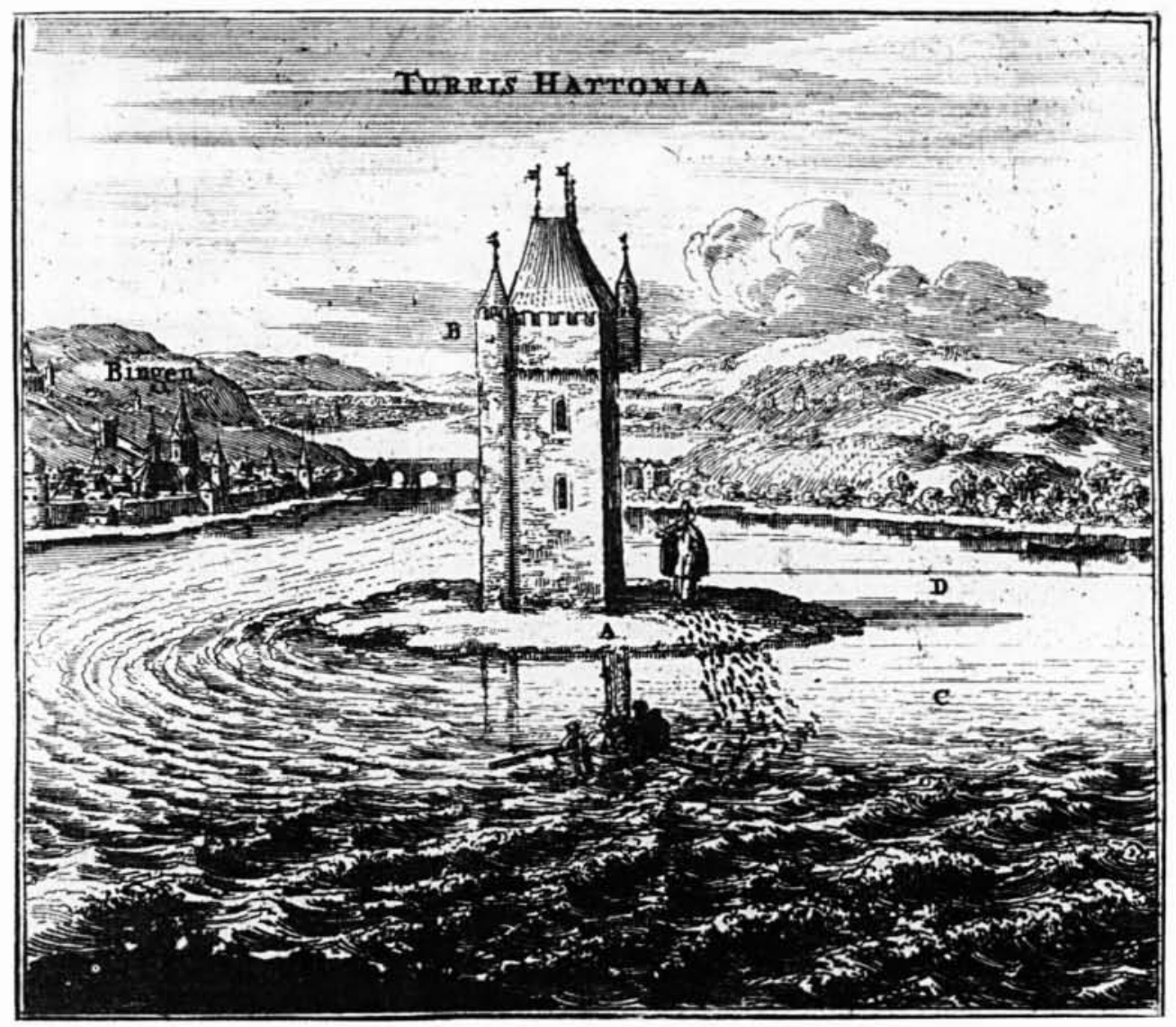

De admirandis mundi cataractis - Jean Herbinius - Amsterdam - 1678 